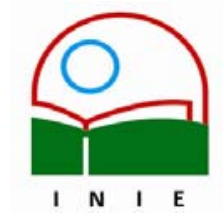

Universidad de Costa Rica

Facultad de Educación

Instituto de Investigación en Educación

ACTUALIDADES INVESTIGATIVAS EN EDUCACION

\title{
EVALUACIÓN DE LA MODALIDAD HÍBRIDA, PRESENCIAL/EN LÍNEA, POR ESTUDIANTES DE POSGRADO EN EDUCACIÓN
}

\author{
EVALUATION OF THE HYBRID EDUCATIONAL FORMULA, PRESENTIAL/ON-LINE, BY \\ GRADUATE STUDENTS IN EDUCATION
}

\author{
Gilles Lavigne ${ }^{1}$ \\ Javier Organista Sandoval ${ }^{2}$ \\ Lucía Coral Aguirre Muñoz
}

\begin{abstract}
Resumen: Durante septiembre de 2002 y enero de 2004, la Maestría en Ciencias Educativas del Instituto de Investigación y Desarrollo Educativo (IIDE) de la Universidad Autónoma de Baja California (UABC) se transformó gradualmente, de una modalidad presencial a una modalidad híbrida (Presencial/En línea). La nueva versión se evaluó en dos ocasiones con la participación de los estudiantes mediante cuestionarios y grupos de discusión. Esta evaluación muestra que el modelo híbrido adoptado, presencial/en línea, fue adecuado ${ }^{4}$. El discurso de los estudiantes muestra que se comprometieron por las bondades de la modalidad, sin embargo siguieron presente problemas tanto didácticos como pedagógicos comunes en los procesos de enseñanza-aprendizaje, además de los problemas inherentes a la modalidad, como el de la tutoría a distancia.
\end{abstract}

Palabras clave: ESTUDIOS DE POSGRADO; CAMBIO TECNOLÓGICO; EDUCACIÓN EN LÍNEA; EVALUACIÓN DE MODELOS; MODERNIZACIÓN EDUCATIVA.

\begin{abstract}
In between September of 2002 and January of 2004, the Instituto de Investigación y Desarrollo Educativo (IIDE) - Universidad Autónoma de Baja California's (UABC) Master in Educational Sciences was put on-line gradually, trimester by trimester. This new version was evaluated twice with the students, through questionnaires and focus groups. The results of this evaluation are presented in this paper; it shows that the hybrid face-to-face/on-line pattern used was appropriate. However the students' discourse shows they involved themselves mainly for practical reasons; it seems clear for them that the usual problems associated with the teaching-learning process, such as didactical and pedagogical one, were not solved by the on-line formula, which moreover displayed new ones, such as tutoring.
\end{abstract}

Key words: GRADUATE STUDIES; TECHNOLOGICAL CHANGE; E-LEARNING; EDUCATIONAL PATTERN EVALUATION; EDUCATION MODERNIZATION.

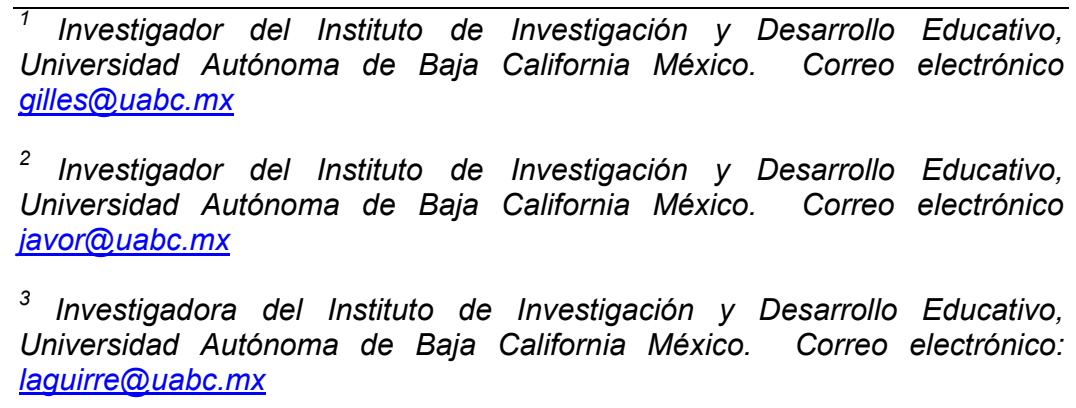

Artículo recibido: 6 de enero, 2006

Aprobado: 27 de abril, 2006 ${ }^{4}$ Partes de este trabajo fueron divulgadas como resultados parciales en congresos internacionales en
2003 y 2004. 


\section{Introducción}

La referencia, hoy común, a la sociedad de la información expresa el impacto del desarrollo de las nuevas tecnologías de la información y de la comunicación (TIC) sobre nuestras sociedades. Al inicio de los noventa, la integración de estas tecnologías en la educación debía favorecer la reconsideración de nuestros métodos de enseñanza y, sobre todo, de aprendizaje. Las esperanzas de renovación de la educación por medio de las TIC, especialmente por el e-learning, ${ }^{5}$ eran variadas y numerosas. Sin duda, Internet nos ofreció un nuevo y fuerte potencial de difusión y adquisición de conocimientos, y los pronósticos eran muy positivos:

The most quoted projections - those made in 2000 by Michael Moe in the Merrill Lynch white paper, The Knowledge Web-boldly proclaimed: Our estimates for the U.S. online market opportunity for knowledge enterprises will grow from \$9.4 billion in 1999 to $\$ 53.3$ billion in 2003 , representing a CAGR [Compound Annual Growth Rate] of 54 percent. At an estimated $\$ 105$ billion, the spending power of college students is huge. Not surprisingly, a growing percentage of their spending is moving online. Currently, students spend $\$ 1.5$ billion online, an amount which is expected to almost triple to $\$ 3.9$ billion by 2002. We estimate that the U.S. market for online higher education alone will grow from $\$ 1.2$ billion in 1999 to $\$ 7$ billion in 2003 (Zemsky y Massy, 2004, p. 3)

Aunque las universidades estadounidenses invirtieron grandes cantidades de dinero, los resultados no estuvieron a la medida de las proyecciones antes mencionadas. Zemsky y Massy (2004) identificaron varios factores para explicar el revés parcial del desarrollo del elearning en las universidades, en particular, el conservadurismo de las instituciones, de los académicos y de los alumnos (Cheese, 2003). De hecho, cuando los estudiantes tienen la posibilidad de elegir entre una formación tradicional y una en línea, quienes eligen la última lo hacen por razones externas al modo de aprendizaje; son estudiantes no tradicionales, de tiempo parcial, trabajadores, que viven fuera de la universidad y que tienen conflictos de horario (Dutton, Dutton y Perry, 2002).

Sin embargo, la puesta en línea de cursos y programas completos de formación ha alcanzado un notable desarrollo. En la actualidad, el número de cursos y programas

\footnotetext{
${ }^{5}$ Detrás del concepto de e-learning, acuñado en Estados Unidos, se encuentran diferentes enfoques de "enseñanza", de la formación profesional "en servicio" en empresas, hasta la educación superior comercializada como negocio. Pero a pesar de esta diversidad, el concepto denota específicamente el enfoque de un aprendizaje a través del uso de una red, incluida Internet, como medio básico de comunicación e información, que puede integrar otros medios. En este sentido, el concepto es muy útil para identificar una modalidad específica de aprendizaje (Pouts-Lajus, 2002).
} 
accesibles en Internet es impresionante, especialmente en lengua inglesa. Además, las universidades están cada vez más conectadas por lo que utilizan este medio de comunicación para difundir total o parcialmente su enseñanza. Aunque el desarrollo técnico de las universidades mexicanas no es tan amplio como el de las universidades estadounidenses, la mayoría tiene una conexión a Internet, tanto dentro de las aulas, como para el uso de sus docentes, y las que ofrecen educación a distancia están cada vez más involucradas con el e-learning (ANUIES, 2003). Considerando lo anterior, se puede decir que el desarrollo de este nuevo enfoque educativo es un elemento de interés, especialmente en universidades e institutos de educación.

Aunque la tendencia hacia una generalización de las tecnologías de comunicación, así como de la comunicación asincrónica en la educación parece irreversible, los componentes de este hecho social todavía son poco conocidos. Cuando se planea trabajar en línea no se pueden subestimar las advertencias planteadas por varios estudiosos como Herbert I. Schiller y Peter McLaren en los Estados Unidos quienes denunciaron y criticaron el imperialismo americano tras el desarrollo de las TIC (Schiller, 1998; McLaren y Farahmandpur, 2001), Armand Mattelart en Francia quien demostró el rasgo ideológico y mitológico del discurso tecnológico (1997), Francisco Sierra en España quien identificó los principios que la educación virtual debe incorporar (1999) o Michel Sénécal en Canadá quien subrayó la necesidad de sostener el principio de la apropiación ciudadana y democrática de las tecnologías (2002), sólo por mencionar algunos. ${ }^{6}$ Existe, de hecho, el peligro de enfrentar un nuevo imperialismo del Norte a través de la globalización del e-learning.

Dentro de la estructura de la economía del saber o de la nueva economía globalizada se concibe a la educación cada vez más como un factor de producción de riqueza con base en el conocimiento y la creatividad. Desde el momento en que la educación está concebida como un bien comercial, la lógica del mercado está presente, con consecuencias posiblemente comparables a las de la producción industrial. Esta nueva clasificación de la educación ha sido posible por el ensanchamiento tecnológico de los medios de comunicación y de información, los cuales han acelerado la industrialización de la educación, diversificando cada vez un poco más los procesos de enseñanza-aprendizaje. ${ }^{7}$

\footnotetext{
${ }^{6}$ Cada uno de estos autores ha publicado muchos trabajos sobre el tema de las TIC, pero como no son el objeto de este artículo, se ha considerado dar solamente algunas referencias.

${ }^{7}$ El desarrollo de la educación a distancia, a partir del modelo británico implantado a fines de los años sesenta, fue el motor de la industrialización de la educación, acelerado fuertemente por el efecto de las TIC.
} 
En el mundo del e-learning, indudablemente, es oportuno informarse de los obstáculos que deben enfrentarse y, sobre todo, de tomar en cuenta la evaluación de los estudiantes hacia la modalidad utilizada. Después de todo, ellos son la "materia prima" de la educación superior y sin ellos nada puede ser. Además, para mantener un papel de difusor autónomo de conocimientos, hay que optimizar los recursos disponibles y producir materiales pedagógicos de calidad.

Con el fin de evitar discusiones acerca de si existen diferencias entre el e-learning y el aprendizaje a distancia o la educación a distancia, Carol A. Twigg (2001) escribió:

A few words about terminology are in order. [...], the terms distance learning, distance education, distributed learning and on line learning are used more or less interchangeably. [...] In any event, the reader should not draw unwarranted conclusions from a particular usage (p. 200).

Actualmente, no hay una taxonomía vigente y aceptada de las varias modalidades de aprender a distancia con las nuevas tecnologías de información y comunicación. Además, el uso de las TIC en la enseñanza llamada tradicional, en campus y presencial, da una imprecisión adicional que complica el trabajo teórico de circunscribir conceptos con sentido. En enero de 2002, el Instituto de Investigación y Desarrollo Educativo (IIDE) de la Universidad Autónoma de Baja California (UABC) decidió transformar su programa de Maestría en Ciencias Educativas (MCE) a una modalidad mixta en la que se incluyeron sesiones presenciales y virtuales, vía Internet y con apoyo de las videoconferencias.

Con base en la información obtenida en la experiencia de la conversión del programa, el propósito de la presente comunicación es:

1) Presentar las condiciones de la puesta en línea del programa de MCE;

2) Analizar los resultados de dos evaluaciones hechas por los estudiantes del programa. Además, se presentan los resultados relativos a las condiciones materiales y prácticas del aprendizaje en línea, obtenidos con un método comparativo durante dos trimestres.

\section{Referente teórico}

Antes de iniciar el proceso de conversión del programa de Maestría hacia la modalidad híbrida, se recopiló información que permitiera dar respuesta a las siguientes interrogantes: ¿Cuál modalidad sería mejor adoptar para responder a las expectativas de los estudiantes, especialmente con relación a su aprendizaje? ¿Qué formación se debía proporcionar a los profesores del Instituto, para su nuevo rol como docente? ¿Cómo organizar la producción de 
los cursos? ¿Cómo evaluar los impactos de la modalidad híbrida al nivel de los estudiantes?

\section{1 ¿Cuales son las condiciones para convertir un programa de posgrado para operar bajo una modalidad híbrida?}

Fue notorio el hecho de que la conversión de programas educativos completos hacia nuevas modalidades ha sido gradual, en gran medida, por los requerimientos técnicos y humanos que dependen del tipo de institución. Trabajar en línea representa una operación compleja y delicada, con muchos factores interrelacionados por considerar, entre los que destacan el nuevo rol del docente, la autonomía del estudiante, el tipo de materiales educativos, uso de vías de comunicación electrónicas, entre otros, que requieren una integración dinámica (Aoki, Fasse y Stowe, 1998; WICHE, 1997, Anderson y Elloumi, 2004).

Una parte de la información disponible está dirigida a aspectos más especializados como el diseño del curso (Cooper, 1999, 2000), el aprendizaje (Downs, Carlson, Repman y Clark 1999), las infraestructuras (Geith y Vignare, 2000), el trabajo docente (Lavigne, 2001), el costo (Cook, 2002; Boettcher, 2004).

De la revisión documental se logra determinar que gran cantidad de información se enfoca más a los resultados, combinados con recomendaciones. Así, se necesita un gran apoyo de la administración, de los académicos y de los estudiantes, además de una planificación paso a paso, sin olvidar un enfoque pedagógico adecuado (Thormann, 1999; Kaminski y Milheim, 2002). Algunas recomendaciones parecen muy importantes, como: mantener una comunicación eficaz y constante con los estudiantes, desarrollar materiales pedagógicos en varios formatos con un nuevo enfoque, capacitar a los académicos, proporcionar una capacitación inicial a los estudiantes (Cooper, 2000; Kupritz, 2000; Levy, 2003).

Sin embargo, la información más interesante se refiere a la evaluación del e-learning, misma que comprende varios temas y está dividida en dos grandes corrientes. Como es un negocio, en parte, se han desarrollado paquetes y servicios especializados respecto a cómo evaluar un producto de e-learning para los consumidores individuales o empresariales. ${ }^{8}$ Además, con la puesta en línea de cursos y programas tanto a nivel licenciatura como en posgrado en universidades y colegios, ha exigido grandes inversiones con o sin financiamiento del Estado; y como los resultados no han sido los esperados, se han desarrollado investigaciones para evaluar los diferentes componentes de este tipo de aprendizaje, con el fin de explicar el ¿por qué? de su casi total fracaso, tanto en los Estados Unidos y Canadá

${ }^{8}$ ¡Existen 61700000 páginas registradas en Google para la evaluación del e-learning! 
como en Europa.

\section{2 ¿Cuál es la opinión de los estudiantes con relación al e-learning?}

Una revisión exhaustiva de la literatura científica sobre el e-learning resulta difícil debido a la multiplicidad de estudios y a la diversidad de los temas vinculados a él. ${ }^{9}$ Además, el campo de la educación es fluctuante y dinámico después de 40 años, primero por la remodelación de la educación a distancia, después por la introducción del audiovisual y de la computación, y ahora por las nuevas tecnologías de información y comunicación. La riqueza de las innovaciones, teóricas, metodológicas y tecnológicas es fenomenal, pero el resultado es que la indeterminación es más grande que nunca. Sin embargo, una revisión, incluso breve, es la única manera de apoyarse antes de sumergirse en el terreno inestable del e-learning.

Los científicos han identificado diferentes conjuntos de variables por evaluar en el e-learning: las del contexto socio-económico, las del ambiente de aprendizaje, las de la tecnología, la de la pedagogía y las de los estudiantes (Hughes y Attwell, 2003). Existen por lo menos tres modelos de evaluación -con más o menos los mismos conjuntos de variables- y dos procedimientos - uno estadounidense y el otro europeo- para implementar un proceso llamado benchmarking, el cual sirve para comparar instituciones obteniendo resultados excelentes de calidad (Rubio, 2003). Por otra parte, se han desarrollado hasta 14 medidas diferentes para evaluar el e-learning (Bonk y Dennen, 2002). Pero, incluyendo todo, se identifican cinco aspectos por evaluar: contenido, búsqueda y extracción de los archivos, acceso, tutoría y manejo (Boonen y Van Petegem, 2003).

Si la evaluación del e-learning tiene como objetivo mejorar el diseño, la organización técnica, el interfaz y la tutoría para facilitar el aprendizaje, se afronta a las mismas dudas y críticas que la educación a distancia en comparación con el aprendizaje presencial, todavía dominante, el cual fue objeto de investigación y de debate. Lo que ahora se puede asegurar es que el aprendizaje es equivalente cuando el apoyo tutorial en modalidad a distancia es eficaz, aunque depende de las materias, porque hay algunas difíciles de enseñar a distancia. (Young, 2000; Kassop, 2004).

En fin, como lo mencionaron Boonen y Van Petegem: "[...] but any quality approach of learning in higher education should keep in mind the final users of learning, i. e. the students." (2003, p. 3).

La posición más generalizada de los estudiantes tiene un sentido ambiguo: por un lado se

${ }^{9}$ Al parecer sólo Comunidad Europea financia 300 proyectos vinculados al e-learning. 
declaran a favor del aprendizaje en línea y, por otro, afirman que les gusta mucho el aprendizaje presencial. Cuando tienen la posibilidad de elegir la modalidad de su aprendizaje prefieren los cursos tradicionales (Cheese, 2003; Mac Keogh, 2003; Dutton, Dutton y Perry, 2002). Otra característica es que los estudiantes en la modalidad en línea no usan las posibilidades ofrecidas por los diseños interactivos con hipertextos, estudian de manera lineal, además prefieren las versiones impresas de los materiales pedagógicos (Picciano, 2002) y cursos muy bien estructurados con lecturas y tareas escritas (Katzowitz y May, 2003).

El análisis de las opiniones y percepciones de los estudiantes ha demostrado correlaciones significativas entre una percepción positiva y varias variables: acceso fácil, constante y eficaz con los tutores; participación en las actividades comunes, como foros; interacción con pares. También el género y la edad pueden influir en la percepción: las mujeres y los estudiantes que tienen más de 35 años demuestran una postura más positiva; mientras que el nivel de habilidad computacional no es un factor influyente (Fredericksen et al, 2000).

Tomando en cuenta el débil éxito del e-learning en el mundo académico, se ha desarrollado una modalidad híbrida, presencial y en línea. Al parecer, esta fórmula ha encontrado la aprobación de muchos académicos y estudiantes, y se ha ampliado rápidamente (Young, 2002).

Por último, es conveniente mencionar que en Internet se encuentran modelos de cuestionarios para hacer una evaluación por los estudiantes acerca del aprendizaje logrado, de la calidad de los materiales educativos, de las interacciones con el tutor, por mencionar algunos. Las rúbricas son más o menos similares, congruentes con los aspectos de investigación señalados en párrafos anteriores, y usan la escala de Likert como técnica de medición (Carliner, 2002; SPOT PLUS, 2004).

\section{Contexto institucional y operativo de la puesta en línea de la maestría}

A partir de febrero de 2002, la dirección del IIDE, tomó las siguientes acciones:

- Otorgar la responsabilidad a la Coordinación de la MCE, como entidad administrativa, para llevar a cabo la conversión.

- Movilizar a un profesor-investigador del instituto, especializado en tecnología multimedia con fines pedagógicos.

- Contratar a un especialista con formación a distancia para participar en la capacitación de los profesores del Instituto, para que actúe como consejero y 
coordine la producción de los cursos, considerando las sugerencias instruccionales de los docentes respectivos.

- Liberar presupuesto específico.

- Tomar la decisión de utilizar la plataforma Web del propio Instituto.

En marzo de 2002, la coordinación de la MCE emprendió las siguientes acciones:

- Contratar a tiempo parcial, a dos becarios universitarios en el área de informática para garantizar la digitalización de los materiales educativos.

- Contratar a una agencia de diseño para la elaboración de la interfase gráfica final y para montarla en el servidor del Instituto.

- Elaborar guías y formatos de conversión, calendario, entre otras herramientas, para el control riguroso de los avances.

Conducir la transformación de la Maestría en Ciencias Educativas (MCE) del IIDE, de una modalidad presencial a una mixta (presencial y en línea), requirió dos años y medio de trabajo técnico y la colaboración de nueve profesores-investigadores del Instituto más un profesor independiente para formalizar los contenidos de los cursos. Cabe destacar que la implementación de los cursos en línea se realizó en paralelo con el avance de los alumnos en el programa, trimestre por trimestre.

Antes de abordar la evaluación de la operación, es importante recordar algunas de las características de la MCE. Ésta contempla la formación de investigadores en el ámbito de la educación. Está dirigida a profesionales de la educación, profesores, administradores, consejeros pedagógicos y quienes desean - por la vía de la investigación - mejorar la calidad de la enseñanza en su medio respectivo.

El nivel de estudio de posgrado y el ámbito de formación en investigación requieren un enfoque didáctico eficaz y diversificado. Dado que previamente se habían impartido los cursos de forma presencial, los profesores ya habían elaborado un marco didáctico y pedagógico específico para cada uno de ellos. Con el fin de respetar el trabajo académico de cada profesor, se les permitió, en primer lugar, establecer a su manera las modalidades de su curso. Se respetó, al mismo tiempo, la matriz organizativa de la información elaborada colegiadamente. En segundo lugar, se les invitó a promover la colaboración y evitar la resistencia identificada en las evaluaciones del e-learning. Es decir, cada profesor podía proporcionar a su gusto las modalidades pedagógicas de su curso a partir de los formatos disponibles, o sea, el presencial, la videoconferencia, el trabajo individual, el trabajo cooperativo, más una serie de herramientas de comunicación en línea como: foros, chat y correo electrónico; sin olvidar los medios más clásicos como la lectura (en formato PDF, 
fotocopias, libros, etc.), diapositivas o video, entre otros. Entonces, a fin de cuentas aunque se trabaje bajo un común denominador, todos los cursos son diferentes.

La matriz organizativa de la información, desarrollada a través de un trabajo colectivo de los académicos del Instituto, se estructura en dos niveles principales separados por una barrera. El primero, público, asume el papel de portal y es accesible a través de la dirección electrónica de la maestría (http://eduweb.ens.uabc.mx). Esta página reúne toda la información relativa a la MCE y permite el acceso a los cursos. El segundo nivel, reservado para los estudiantes y profesores, estructura la información inherente a la formación. La matriz organizativa define cinco tipos de información válidos para todos los cursos: "Información general", "Actividades", "Herramientas", "Comunicación" y "Ayuda" (ver Figura 1).

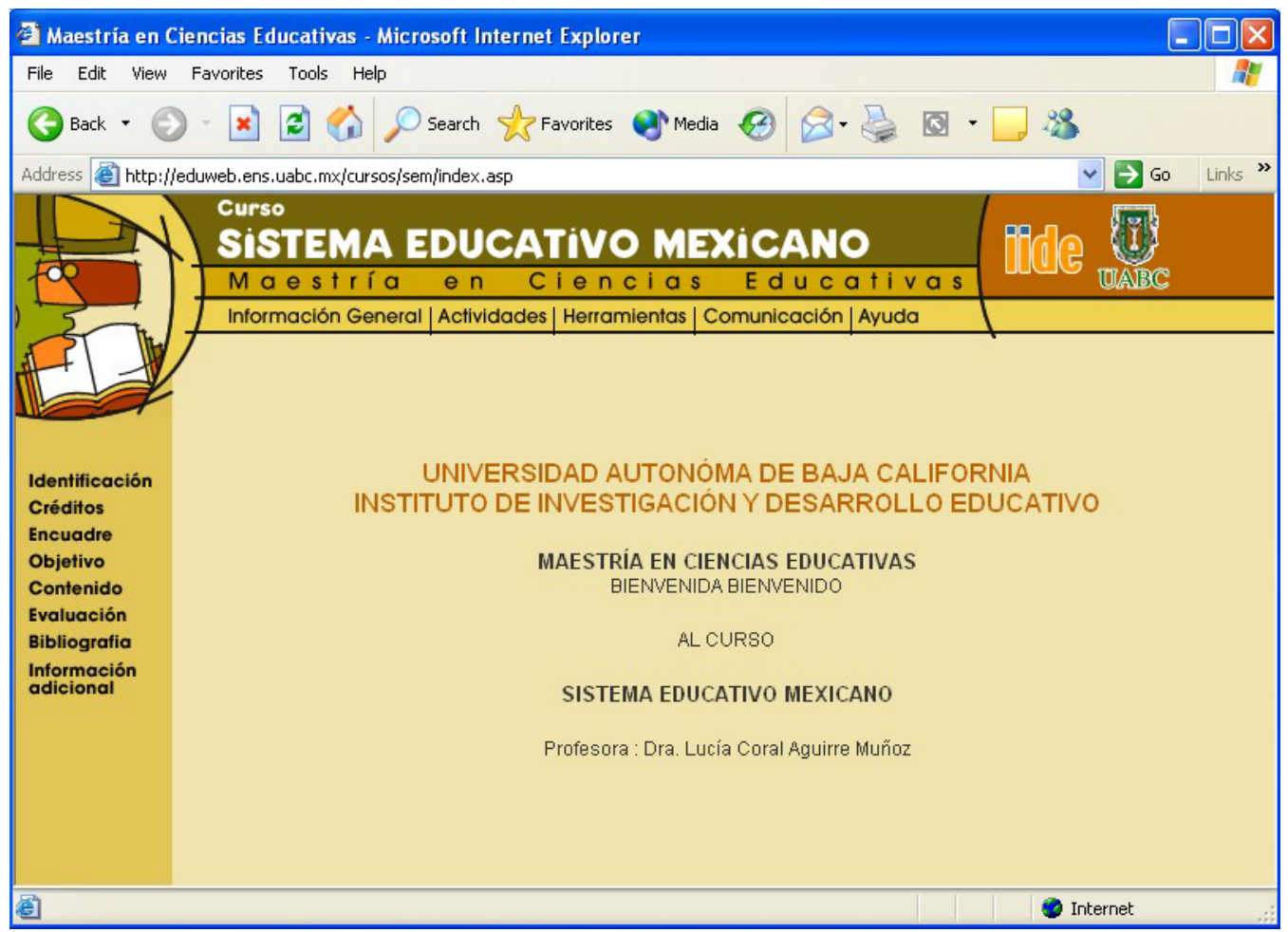

Figura 1. Pantalla principal típica de inicio en un curso formal del programa de MCE

En lo que se refiere a aspectos didácticos y pedagógicos, los profesores decidieron mantener el enfoque por sesión. Esta elección respondió a la preocupación, por una parte, de mantener un seguimiento de los avances de los estudiantes y, por la otra, de conservar como base el marco didáctico elaborado para los cursos presenciales. Esta decisión se apega a los resultados de las investigaciones que muestran que los estudiantes prefieren cursos bien estructurados y en los que puedan estudiar de manera lineal. 


\section{Metodología de evaluación}

Para tener la opinión y la percepción de los estudiantes de la maestría acerca de la modalidad presencial/en-línea, se realizó una evaluación interna cuantitativa y cualitativa por medio de dos técnicas, la del cuestionario y la del grupo de discusión.

La evaluación, cuya primera etapa se llevó a cabo en diciembre de 2002 y la segunda en abril de 2003, respondió a los siguientes objetivos:

1) Identificar los impactos educativos, tanto positivos como negativos de la modalidad híbrida utilizada.

2) Identificar los problemas técnicos que se presentaron, de tal modo que sea posible corregirlos y así mejorar la siguiente impartición de los cursos.

\subsection{Marco de análisis}

Con el fin de solucionar lo más adecuadamente posible los problemas de esta evaluación, el equipo responsable del proyecto produjo un modelo teórico que respondía a su percepción y comprensión del planteamiento que debía realizarse. A partir de este modelo, se obtuvieron las principales dimensiones que debían estructurar la evaluación (ver Tabla 1): los medios (como los técnicos y de aprendizaje), la amplitud (de aprendizaje, del horario), la interacción (con el material, los profesores, los compañeros), los componentes técnicos (habilidades, problemas, eficacia).

Tabla 1: Componentes de la evaluación

\begin{tabular}{|c|c|c|c|c|}
\hline \multirow{2}{*}{ Datos } & \multicolumn{4}{|c|}{ Estudiantes } \\
\hline & Tipo & & mas & Tipo \\
\hline \multirow{2}{*}{$\begin{array}{l}\text { LA FORMULACIÓN } \\
\text { - } \quad \text { Contenido } \\
\text { - } \quad \text { Tareas }\end{array}$} & GD & \begin{tabular}{ll}
\multicolumn{2}{l}{ Aprendizaje } \\
- & Responsabilidad \\
- & Autonomía \\
- & Control
\end{tabular} & 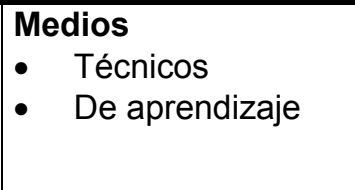 & C \\
\hline & GD & $\begin{array}{l}\text { Objetivos } \\
\text { - } \quad \text { De aprendizaje } \\
\text { - } \quad \text { Personales }\end{array}$ & $\begin{array}{l}\text { Tamaño } \\
\text { - } \quad \text { Para aprender } \\
\text { - } \quad \text { Del horario }\end{array}$ & C \\
\hline $\begin{array}{ll} & \text { El formato } \\
\text { - } & \text { Presencial } \\
\text { - } & \text { Videoconferencia } \\
\text { - } & \text { En línea }\end{array}$ & GD & $\begin{array}{l}\text { Comunicación } \\
\text { - Con profesores } \\
\text { - Con } \\
\text { compañeros }\end{array}$ & $\begin{array}{l}\text { Interacción } \\
\text { - } \quad \text { Con el material } \\
\text { - } \quad \text { Con profesores } \\
\text { - } \quad \text { Con compañeros }\end{array}$ & C \\
\hline
\end{tabular}




\begin{tabular}{|c|c|c|c|c|}
\hline $\begin{array}{ll}\text { - } & \text { Pdf } \\
\text { - } & \text { Correo electrónico } \\
\text { - } & \text { Foro } \\
\text { - } & \text { Horario }\end{array}$ & GD & $\begin{array}{l}\text { Encuadre } \\
\text { - }\end{array}$ & 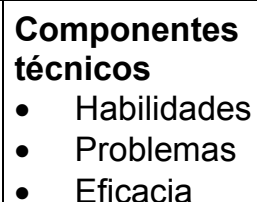 & C \\
\hline
\end{tabular}

GD = Grupo de discusión; C = Cuestionario

\subsection{Procedimiento metodológico}

\subsubsection{Los cuestionarios}

Al considerar lo anterior, se elaboró un cuestionario con 95 reactivos para la primera etapa y 100 para la segunda. ${ }^{10}$ Los estudiantes inscritos al programa de maestría a partir de septiembre de 2002 eran 20. El primer cuestionario se administró a 15 de ellos, en diciembre de 2002 y el segundo se aplicó a 12 estudiantes en abril de 2003.

El procedimiento por cuestionario aplicado proporcionó datos cuantitativos que se analizaron con herramientas de estadística descriptiva. La evaluación comprendió siete cursos. Los resultados aquí presentados sólo describen las diferencias observadas entre las dos fases de la evaluación, organizados según la formulación del cuestionario.

Con el fin de simplificar la lectura, la mayoría de las preguntas planteadas piden una respuesta dentro de una escala ordinal de Likert. De acuerdo con las respuestas efectivas del número máximo posible, se hizo una estimación ponderada expresada en porcentaje. Este porcentaje dio cuenta del nivel de satisfacción, eficacia o facilidad, expresado por los estudiantes, según la declaración de la cuestión. Las diferencias entre las dos medidas se interpretaron en puntos, ganados o perdidos.

\subsubsection{Los grupos de discusión}

Además, se organizaron dos grupos de discusión, paralelamente a la administración de los cuestionarios. En el primero participaron 12 estudiantes y 6 en el segundo. La guía de discusión estuvo estructurada con los mismos componentes de los cuestionarios. El primer grupo fue dirigido por un profesor y el segundo fue por un estudiante. Las discusiones duraron, más o menos, una hora y media por sesión y fueron grabadas en audio. El primer grupo de discusión se realizó el mismo día que el cuestionario, el segundo, dos semanas antes de administrar el cuestionario.

El contenido de los grupos de discusión fue tratado con un análisis de contenido de tipo

\footnotetext{
${ }^{10}$ La mayoría de los reactivos se destinaron a la evaluación de los cursos; los resultados de estas evaluaciones no son presentados en este artículo.
} 
temático. En el caso del primer grupo, el análisis tuvo como unidad de anotación, el tema y como contexto, el parágrafo. Para el segundo, la unidad fue la palabra y la oración fue el contexto. En ambos casos, el tema fue la unidad de significación. Se optó por desarrollar un procedimiento mediante las funciones del programa Word (MS Office) para el análisis del segundo grupo.

\section{Resultados y discusión}

\subsection{Los cuestionarios}

La mayoría de los estudiantes participantes no tenía experiencia previa con cursos a distancia; era la primera vez que los conocían.

Las principales observaciones encontradas fueron, que:

- Los estudiantes prefieren la distancia ( $83.8 \%$ y $77.1 \%$, por cada trimestre).

- Les fue difícil organizar su trabajo durante el primer trimestre (68.3\%), pero aprendieron a hacerlo durante el segundo trimestre (75.0\%). Esta observación coincide con observaciones similares en situaciones comparables (Karsenti et al., 2002).

- La mayoría de los estudiantes modificó su organización del trabajo para volverla más flexible.

- Aumentaron el tiempo de estudio entre los dos trimestres, pero la valoración que hicieron siguió siendo igual.

- Los estudiantes mejoraron sus habilidades de comunicación.

- Los estudiantes apreciaron el correo electrónico como medio de comunicación.

- Todos los estudiantes desarrollaron nuevas habilidades y la mayoría de ellos consideró poseer las necesarias para seguir participando en cursos en línea.

- La mayoría de ellos consideró suficiente la capacidad de su equipo de cómputo.

Lo que es importante notar aquí (ver Tabla 2), es que los estudiantes aprendieron a estudiar a distancia durante los dos primeros trimestres del primer año de la maestría. Se adaptaron y cambiaron sus costumbres en cuanto al lugar de estudio como al del tiempo que dedicaban; adoptaron prácticas más flexibles; trabajaron más el segundo trimestre, como resultado de la responsabilidad propia de su aprendizaje y de su autonomía para organizar su trabajo. De esta manera, no es una sorpresa que la educación a distancia no les agrade tanto en la segunda evaluación. 
Tabla 2. Organización y amplitud del trabajo de los estudiantes

\begin{tabular}{|c|c|c|c|}
\hline \multirow[b]{2}{*}{ Enunciado } & \multicolumn{3}{|c|}{ Media } \\
\hline & $\begin{array}{l}\text { Diciembre } \\
1^{\circ} \text { evaluación }\end{array}$ & $\begin{array}{c}\text { Abril } \\
2^{\circ} \text { evaluación }\end{array}$ & Variación \\
\hline $\begin{array}{l}1.1 \text { ¿Cuál es tu apreciación de recibir una } \\
\text { educación a distancia? }\end{array}$ & 83.3 & 77.1 & -6.2 \\
\hline $\begin{array}{l}2.1 \quad \text { ¿Lograste organizar tus tiempos de } \\
\text { trabajo para la Maestría? }\end{array}$ & 68.3 & 75.0 & +6.7 \\
\hline $\begin{array}{l}\text { 2.2 De manera general, ¿en qué momento } \\
\text { del día trabajaste? } \\
\text { - Por la mañana } \\
\text { - Por la tarde } \\
\text { - Por la noche } \\
\text { - En horario indistinto }\end{array}$ & $\begin{array}{r}7.0 \\
7.0 \\
67.0 \\
20.0\end{array}$ & $\begin{array}{r}0.0 \\
8.0 \\
67.0 \\
25.0\end{array}$ & $\begin{array}{r}-7.0 \\
+1.0 \\
0.0 \\
+5.0\end{array}$ \\
\hline $\begin{array}{l}2.3 \text { De manera general, ¿en qué lugar } \\
\text { estudiaste? } \\
\begin{array}{c}\text { - Oficina } \\
\text { - Hogar } \\
\text { - Varios }\end{array}\end{array}$ & $\begin{array}{l}33.0 \\
53.0 \\
13.0\end{array}$ & $\begin{array}{r}8.0 \\
42.0 \\
50.0\end{array}$ & $\begin{array}{r}-25.0 \\
-11.0 \\
+37.0\end{array}$ \\
\hline $\begin{array}{l}\text { 4.1 En promedio, ¿cuánto tiempo semanal } \\
\text { dedicaste a la preparación de cada sesión? } \\
\text { - A estudiar } \\
\text { - A las lecturas } \\
\text { - A las tareas } \\
\text { - A los foros }\end{array}$ & $\begin{array}{r}5.7^{*} \\
6.6 \\
3.4 \\
1.6\end{array}$ & $\begin{array}{r}7.3^{*} \\
8.3 \\
7.1 \\
2.2\end{array}$ & $\begin{array}{l}+1.6 \\
+1.7 \\
+3.7 \\
+0.6\end{array}$ \\
\hline $\begin{array}{l}4.2 \text { ¿Cómo evalúas la amplitud del tiempo } \\
\text { exigido en total para tu aprendizaje? }\end{array}$ & 70.0 & 69.5 & -0.5 \\
\hline 7.1 ¿Te comunicaste con tus compañeros? & 100.0 & 100.0 & 0.0 \\
\hline
\end{tabular}

${ }^{*}$ Nota: ¡En horas!

Una segunda observación de importancia es que los estudiantes desarrollaron la costumbre de comunicarse. Aunque el programa de maestría no tiene ninguna dimensión de trabajo colaborativo, los estudiantes decidieron compartir sus experiencias de aprendizaje, sus problemas, sus éxitos.

En la Tabla 3 se puede observar que los estudiantes no tuvieron problemas con el correo electrónico, y sí han manifestado reticencias para enviar sus trabajos por correo electrónico; tal vez esto se debe a que los profesores involucrados en el segundo trimestre no contestaron tan rápidamente que los del primer trimestre. Esta es una crítica muy fuerte de los estudiantes, ya que sabemos que el tiempo de respuesta del tutor es una característica básica en el apoyo pedagógico que debemos dar a los estudiantes que se forman a distancia. 
Tabla 3. Apoyo a los estudiantes

\begin{tabular}{|l|c|c|c|}
\hline \multicolumn{1}{|c|}{ Enunciado } & \multicolumn{3}{c|}{ Media } \\
\cline { 2 - 4 } & $\begin{array}{c}\text { Diciembre } \\
\mathbf{1}^{\circ} \text { evaluación }\end{array}$ & $\begin{array}{c}\text { Abril } \\
\mathbf{2}^{\circ} \text { evaluación }\end{array}$ & Variación \\
\hline $\begin{array}{l}\text { 6.1 ¿Cuál es tu apreciación del correo } \\
\text { electrónico como medio de comunicación } \\
\text { principal? }\end{array}$ & 70.0 & 77.1 & +7.1 \\
\hline $\begin{array}{l}\text { 6.3 ¿Te gustó la utilización del correo } \\
\text { electrónico para transmitir tus trabajos? }\end{array}$ & 88.3 & 75.0 & -13.3 \\
\hline $\begin{array}{l}\text { 6.2 ¿Cuánto tiempo tomó el profesor } \\
\text { responsable del curso para contestarte? }\end{array}$ & & & \\
- Menos de 24 h & 61.9 & 38.2 & -23.7 \\
- En 24 h & 14.7 & 32.4 & +17.7 \\
- En 48 h & 5.9 & 5.9 & 0.0 \\
- Más de 48 h & 17.7 & 23.5 & +5.8 \\
\hline
\end{tabular}

Es interesante notar en la Tabla 4 que los estudiantes desarrollaron nuevas habilidades técnicas en el uso de su equipo de cómputo, sobre todo, en el segundo trimestre. Esta adquisición se agrega al desarrollo de nuevas habilidades para estudiar y aprender a distancia. En este sentido, el hecho de que la maestría estuviera en línea provocó que los estudiantes de esta cohorte adquirieran conocimientos fuera del programa en sí mismo, pero muy útiles en el campo de la investigación.

Tabla 4. Componentes técnicos

\begin{tabular}{|c|c|c|c|}
\hline \multirow[b]{2}{*}{ Enunciado } & \multicolumn{3}{|c|}{ Media } \\
\hline & $\begin{array}{l}\text { Diciembre } \\
1^{\circ} \text { evaluación }\end{array}$ & $\begin{array}{c}\text { Abril } \\
2^{\circ} \text { evaluación }\end{array}$ & Variación \\
\hline $\begin{array}{l}8.1 \text { ¿En qué medida, consideras que } \\
\text { contabas con las habilidades necesarias } \\
\text { para seguir el curso? }\end{array}$ & 63.3 & 66.7 & +3.4 \\
\hline $\begin{array}{c}8.2 \text { ¿Desarrollaste nuevas habilidades? } \\
\text { - Sí } \\
\text { - No }\end{array}$ & $\begin{array}{l}80.0 \\
20.0\end{array}$ & $\begin{array}{r}100.0 \\
0.0 \\
\end{array}$ & $\begin{array}{l}+20.0 \\
-20.0\end{array}$ \\
\hline $\begin{array}{c}9.1 \text { ¿Tuviste problemas de carácter técnico? } \\
- \text { Sí } \\
- \text { No }\end{array}$ & $\begin{array}{l}67.0 \\
33.0\end{array}$ & $\begin{array}{l}58.3 \\
41.7\end{array}$ & $\begin{array}{r}-8.7 \\
+8.7 \\
\end{array}$ \\
\hline $\begin{array}{l}10.1 \text { ¿Consideras la infraestructura del IIDE } \\
\text { suficiente para este tipo de formación? }\end{array}$ & 85.0 & 72.9 & -12.1 \\
\hline $\begin{array}{l}10.2 \text { ¿Consideras tu equipo de cómputo } \\
\text { adecuado para apoyarte con este tipo de } \\
\text { formación? }\end{array}$ & 63.3 & 77.1 & +13.8 \\
\hline
\end{tabular}




\subsection{Los grupos de discusión}

\subsubsection{Primer grupo de discusión}

En general, los estudiantes consideraron muy difícil organizar su trabajo por sí mismos; pero con el tiempo y con la experiencia adquirida, el estudio se ha transformado en un trabajo más fácil y más eficaz para ellos. Destacaron que los intercambios entre ellos les ayudaron a asumir su trabajo de aprendizaje. Solicitaron, incluso, que el Instituto desarrolle actividades de trabajo cooperativo. Los comentarios obtenidos permiten considerar que la socialización a través de la relación entre pares, desempeñó un papel importante en la adaptación a la nueva modalidad.

La variable clave en la organización del aprendizaje a distancia, así como del control adquirido, sigue siendo la gestión del tiempo. Tomando en cuenta la programación intensa de las actividades de aprendizaje, los estudiantes cuestionaron el control de su aprendizaje. Pero consideraron este tipo de programación como una ayuda y no como una obligación, después de tomar en cuenta, que si el programa de la MCE no se hubiera ofrecido a distancia la mayoría de ellos no hubiese podido inscribirse, debido a sus obligaciones profesionales y familiares, y a su lugar de vivienda.

Así, los estudiantes manifestaron que les agradó la modalidad de aprendizaje a distancia, aunque con el deseo de contar con encuentros presenciales. En este sentido, Las videoconferencias, cuando no hay problemas técnicos, son un sustituto aceptable.

Por otra parte, en lo que se refiere a los foros y al chat, los estudiantes no los consideran como medios eficaces de aprendizaje, según ellos son únicamente apoyos. No obstante, aceptaron la utilización de diapositivas y videos, y no plantearon ningún problema técnico. Resultó también claro que la utilización del correo electrónico fue muy aceptada, aunque los estudiantes solicitan a los profesores que respondan rápidamente a sus envíos, trabajos o peticiones particulares.

De manera sintética, los estudiantes parecen haber recorrido una ruta que implicaría cuatro etapas (ver Figura 2).

Primera etapa: la clase presencial. Todos reconocieron haber abordado su formación a partir de la única experiencia conocida, la de la clase magistral, donde el profesor controla todo y da todas las instrucciones para la sesión siguiente. Bastaba con estar presente y con seguir las instrucciones.

Segunda etapa: el desconcierto. Después de la primera sesión, realizada presencialmente, todos conocieron una fase de desasosiego, caracterizada por la obligación de organizarse a sí mismos para estudiar, sin saber cómo hacerlo. 
Tercera etapa: una nueva eficacia. Bajo la influencia de dos factores externos, o sea, la adquisición de una experiencia y la socialización dentro el grupo por los intercambios entre colegas, se realizó otra etapa marcada por el desarrollo de una eficacia a organizarse. El aprendizaje se convirtió en un trabajo, si no rutinario, al menos regular. Es cuando apreciaron la distancia.

Cuarta etapa: el control. Al término del trimestre alcanzaron una última etapa calificada como de control y crítica. Se dieron cuenta, de hecho, de que la programación de la formación era demasiado estricta para poder controlar realmente su aprendizaje. Por el contrario, reconocieron que esta misma programación, intensa, les permitió abarcar la materia a tiempo, garantizándoles un aprendizaje adecuado. Punto fundamental, de acuerdo con ellos, fue la necesidad de intercambios entre estudiantes, garantizados por las sesiones presenciales y las videoconferencias.

Más allá de la contradicción aparente entre la solicitud de cierta flexibilidad para promover una autonomía más real y el reconocimiento de los beneficios de una programación intensa en términos de aprendizaje, es decir, entre las ventajas de la distancia y las de la presencia, es posible encontrar los límites que se deben respetar para el diseño de cursos híbridos.

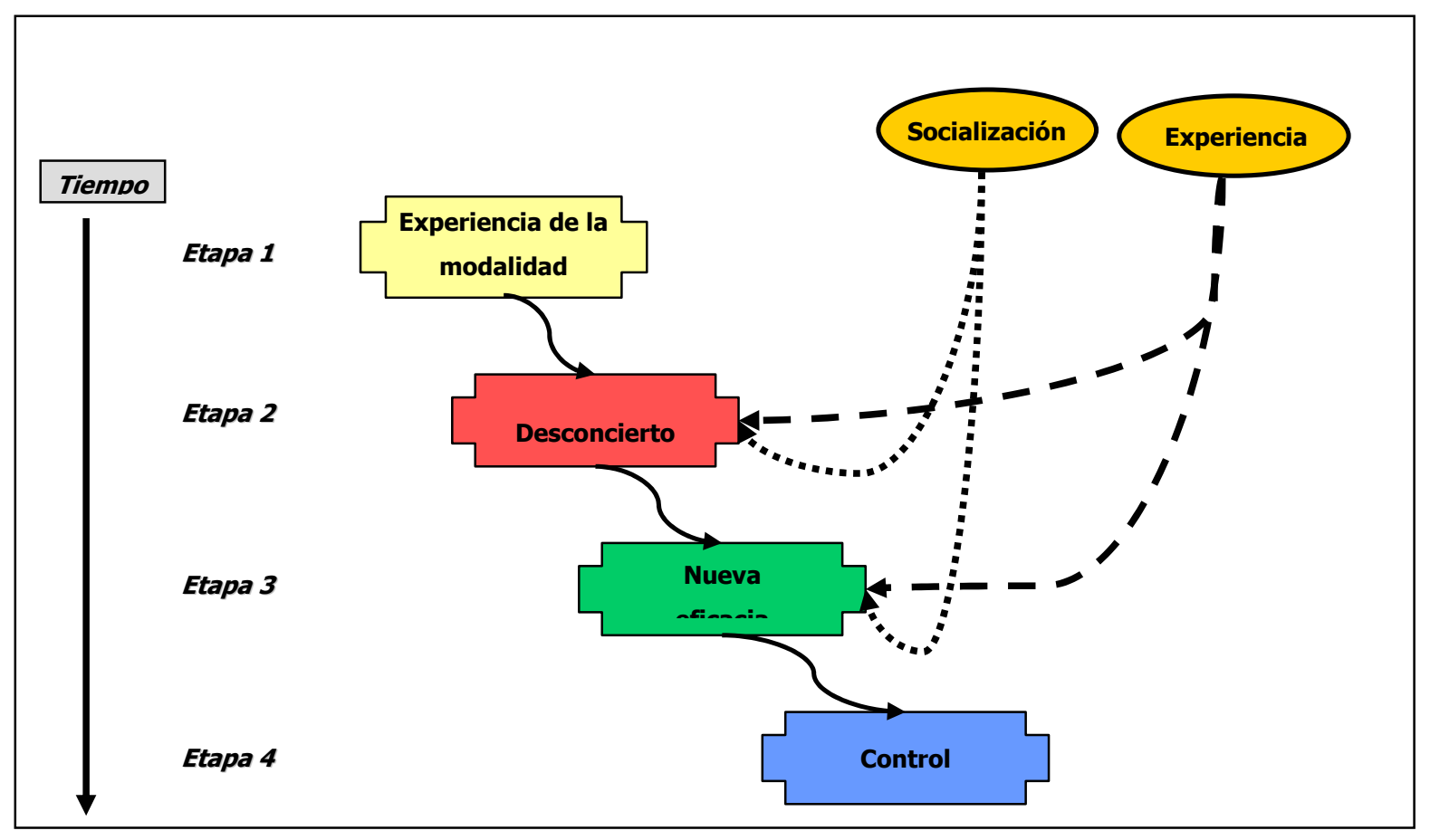

Figura 2. Representación de la discusión del primer grupo 


\subsubsection{Segundo grupo de discusión}

Las discusiones en el segundo grupo de discusión se focalizaron en el aprendizaje a distancia o en línea. Se obtuvo una percepción muy madura, objetiva y compleja. Al centro de la figura 3, se presenta un triple juicio: i) aprender en línea o a distancia ${ }^{11}$ es bueno, ii) no es fácil y iii) requiere mucho trabajo. A partir de estos juicios, se puede reconocer en los discursos de los participantes secuencias explicativas de cada afirmación.

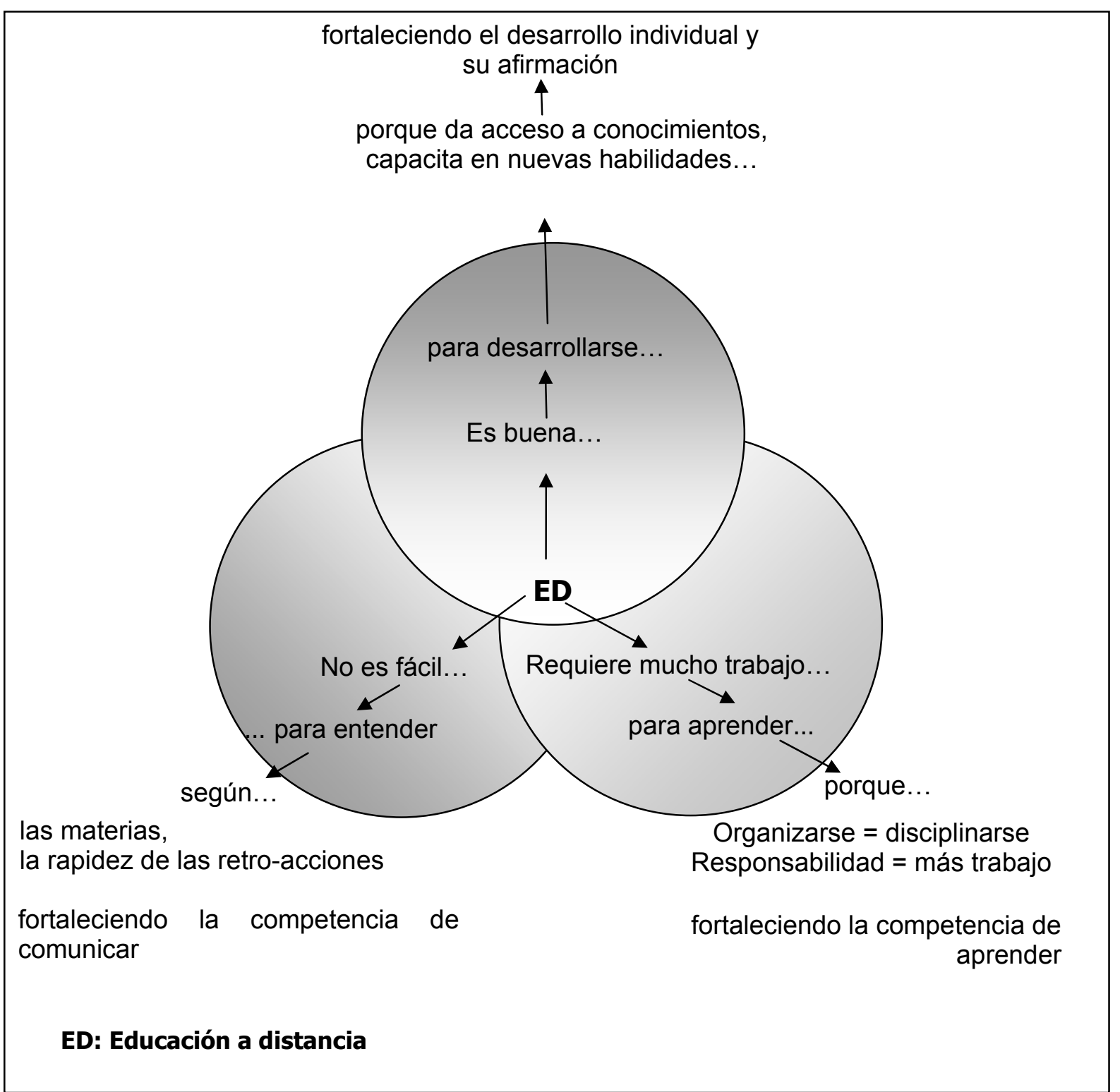

Figura 3: Síntesis de la percepción del aprendizaje a distancia por los estudiantes

\footnotetext{
${ }^{11}$ Como esta experiencia de educación en línea fue la primera experiencia de los estudiantes de esta cohorte, para ellos los conceptos "en línea" o "a distancia" tuvieron el mismo sentido y los utilizaron sin distinción.
} 
Aprender en línea requiere mucho trabajo porque la responsabilidad del estudiante amplía la necesidad de trabajar más para aprender, además de organizar su estudio mediante una disciplina muy severa. Esto se realiza por el tratamiento del material pedagógico según un horario; es decir, la integración de las lecturas, las actividades y la interacción de acuerdo con las fechas límite, con el fin de aprender. Entonces, en resumen, hay dos tipos de trabajos: uno para organizar el aprendizaje y, el otro, el aprendizaje mismo. Un impacto reconocido del trabajo asociado a la modalidad en línea es fortalecer la organización del trabajo que permite la construcción de conocimientos.

Aprender en línea no es fácil, porque el entendimiento de los contenidos se caracteriza por una dificultad variable según las materias y, aún peor, por la rapidez también variable de las respuestas de los profesores a las dudas. De hecho, en caso de duda, como los estudiantes están solos, la retroalimentación de los profesores es casi la única manera de guiar la comprensión. La comunicación y la interacción facilitan el entendimiento; cuando hay, por ejemplo, foros o chat se favorece el intercambio de dudas, ideas, información con el profesor o con los colegas. Un impacto de la dificultad de aprender a distancia es el de promover el intercambio, la cooperación, la comunicación; es decir, la competencia de comunicar.

La modalidad de aprendizaje en línea es buena porque da acceso a una formación de otro modo inaccesible. Además de proveer conocimientos, esta modalidad favorece el desarrollo de habilidades técnicas como también organizacionales y de comunicación, las cuales son también aportaciones para los individuos. Entonces, el aprendizaje a distancia se apoya en una capacitación práctica que contribuye al desarrollo personal de los estudiantes y a su afirmación.

Las percepciones de los estudiantes de la MCE del IIDE se inscriben en la esfera de las observaciones realizadas en otras investigaciones sobre e-learning. El hecho que la MCE se difundió en modalidad híbrida, en línea y presencial, que el diseño de los cursos privilegió una estructura muy intensa, que el uso de hipertextos e hipervínculos se limitó a lo esencial y directamente útil, que la marcha pedagógica elegida ha imitado la de los cursos presenciales, todo esto ha reducido el nivel de novedad, favoreciendo una aceptación mayor del aprendizaje en línea por los estudiantes. Sin embargo, estas percepciones y opiniones de los estudiantes subrayan las variables claves de la problemática del aprendizaje a distancia o en línea. 


\section{Conclusiones}

Al nivel de los impactos educativos se concluyó que la puesta en modalidad híbrida de la maestría no generó condiciones negativas, no hay duda de que los estudiantes de la Maestría en Ciencias Educativas del IIDE eligieron continuar con esta formación de modalidad mixta, parte presencial $( \pm 20 \%)$ y parte a distancia o en línea $( \pm 80 \%)$. Aunque con incertidumbre en el primer trimestre, aprendieron a organizar su trabajo. Los estudiantes consideraron la modalidad mixta adecuada y alcanzaron un aprendizaje equivalente. Refirieron la importancia de contar con los materiales en línea, lo que les permitió flexibilidad de horario de lecturas y avances, de acuerdo con la carga laboral de cada participante.

Al final del segundo trimestre de la formación con esta modalidad, los estudiantes adquirieron una percepción muy clara de la modalidad en línea y fueron capaces de emitir juicios muy profundos. Sin duda alguna, la conversión de programas o de cursos a distancia no puede hacerse sin tomar en cuenta los componentes didácticos y pedagógicos del aprendizaje, por sí mismos y en línea. Dicho de otro modo, adaptar la modalidad de educación en línea no es un trabajo de técnicos, y los académicos deben involucrarse para mantener como primer factor la pedagogía.

En perjuicio de muchos agentes gubernamentales y muchas instituciones, varios estudios señalan que los cuerpos académicos y los estudiantes encuestados manifiestan reticencias que no parecen ser solamente el resultado de algún conservadurismo. En efecto, como lo muestra este estudio, si los estudiantes están de acuerdo con la educación en línea, lo están por pragmatismo y de ningún modo se dejan engañar por las debilidades de la modalidad. Sus compromisos proceden de una evaluación costo-beneficio personal.

Por último, los discursos de los estudiantes han devuelto el análisis al fundamento mismo de los procesos de enseñanza y aprendizaje: la didáctica de las materias, las relaciones pedagógicas, el aprendizaje y el trabajo, entre otros temas. La modalidad en línea no soluciona ni reduce los problemas a los cuales se enfrenta la educación, al contrario, hasta cierto punto, los exacerba.

En lo técnico, los estudiantes no tuvieron problemas, consideraron tener un equipamiento suficientemente adecuado para seguir una formación en línea, y si ellos no poseían todas las habilidades necesarias al principio, las adquirieron después. Es importante señalar que uno de los medios privilegiados de comunicación de los estudiantes fue el correo electrónico.

En cuanto al desarrollo de capacidades particulares en los estudiantes, tanto a nivel de tecnología informativa como de personalidad, ellos mencionan capacidades en cuanto a disciplina, autonomía, etcétera; características que la pedagogía a distancia se atribuye. Sin 
embargo, éstas también pueden desarrollarse en el marco de la enseñanza-aprendizaje tradicional. La diferencia entre las dos fórmulas es que la adquisición de estas habilidades por los estudiantes es una obligación para asegurar la eficiencia de la educación en línea, no así en el caso en la educación tradicional. Desde este punto de vista, la educación en línea apoya el desarrollo personal.

A un nivel más general la experiencia adquirida durante el desarrollo de los cursos en modalidad mixta en un programa formal de maestría, nos sugiere un amplio panorama de aplicación en programas similares en la Universidad Autónoma de Baja California. Son muchos los beneficios de utilizar modalidades alternas, como ya se señaló. Es importante también notar que el cambio a una modalidad casi totalmente en línea ${ }^{12}$ se realizó con un presupuesto muy bajo en comparación con los gastos identificados en los estudios realizados en países del Norte, además, de acuerdo con un calendario muy intenso y muy corto. Esto significa que es posible de apropiarse las nuevas tecnologías según nuestros recursos y con nuestras finalidades.

La enseñanza-aprendizaje en línea no ha generado, hasta ahora, ningún enfoque nuevo de la educación, de hecho, retoma aspectos del constructivismo. En cambio, exige una revisión de fondo de los conceptos y procesos educativos en relación con las teorías de la comunicación y de la información, relativizando un poco el peso teórico de la psicología como único fundamento de las ciencias de la educación. Un estudio como este reactiva perspectivas abandonadas, como la didáctica. La tecnología educativa no puede, como por magia, mediatizar las materias sin tener en cuenta la naturaleza de los contenidos. De la misma manera, la formación en pedagogía no puede sustituirse por la formación en las materias a enseñar. Las dos formaciones son indispensables, y mucho más cuando se trata de enseñanza-aprendizaje en línea.

No obstante, como esta corriente técnico-pedagógica continuará creciendo, sería oportuno utilizarla para renovar la enseñanza, y no solamente en el ámbito del aprendizaje, sino también en la didáctica. Hasta ahora, muchas investigaciones en educación se centran en un proceso de aprendizaje en el que se establece una analogía del estudiante como consumidor, buscando cómo empaquetar el conocimiento de manera que sea asimilable. Por otra parte, no hay muchas investigaciones sobre los contenidos en sí mismos, ni siquiera

\footnotetext{
${ }^{12}$ Los cursos de la MCE del IIDE podrían difundirse totalmente en línea, ha sido por decisión de cada unos de los profesores que se han difundido en modalidad mixta.
} 
como productos. Sin embargo, las nuevas tecnologías de comunicación y de información exigen una revisión de cómo organizar el contenido de las materias a enseñar, utilizando los conceptos propios de las teorías de la comunicación y de la información, y no solamente los de la psicología o, peor, los del marketing. 


\section{Referencias}

Anderson, Terry. y Elloumi, Fathi. (Eds.) (2004). Theory and practice of online learning. On-line Book. Arthabaska: Arthabaska University. Recuperado el 25 de agosto de 2004, de http://cde.athabascau.ca/online book/

Aoki, Kumiko, Fasse, Richard, \& Stowe, Sonny. (1998). A Typology for Distance Education: Tool for Strategic Planning. Recuperado el 24 de agosto de 2004, de ERIC Documento

ED428649 http://search.epnet.com/login.aspx?direct=true\&db=eric\&an=ED428649\&loginpage=login. asp\&site=ehost

Asociación Nacional de Universidades e Instituciones de Educación Superior. (2003). Estudio sobre el uso de las tecnologías de comunicación e información para la virtualización de la educación superior en México. México: ANUIES.

Boettcher, Judith V. (2004). Online course development: What does it cost? Recuperado el 25 de agosto de 2004, de http://www.campus-technology.com/article.asp?id=9676

Bonk, Curt y Dennen, Vanesa. (2002). E-learning evaluation measures. Recuperado el 24 agosto de 2004, de http://www.trainingshare.com/download/train2002/eval methods.doc

Boonen, Annemie y Van Petegem, Wim. (2003). Seequel: Quality in e-learning higher education. Recuperado el 24 agosto de 2004, de http://www.europace.org/services/projects/seequel/Quality in Higher Education.pdf

Carliner, Saul. (2002). Tips for conducting Level 1 evaluations of asynchronous ecourses. What works. Recuperado el 30 de septiembre de 2004, de http://saulcarliner.home.att.net/oll/levelone.htm

Cheese, Peter. (2003). How do learners define blended learning? Recuperado el 6 de octubre de 2004, http://www.Itimagazine.com/ltimagazine/article/articleDetail.jsp?id=52852

Cook, Julian (2002). Evaluating learning technology resources. Recuperado el 24 de agosto de 2003, de http://www.Itsn.ac.uk/application.asp?app=resources.asp\&process=full record\&section=g eneric\&id $=173$

Cooper, Linda (1999). Anatomy of an on-line course. Recuperado el 23 de enero de 2002, de http://www.thejournal.com/magazine/vault/A2071.cfm

Cooper, Linda (2000). On-line courses: Tips for making them work. Recuperado el 23 de enero de 2002, de http://www.usq.edu.au/electpub/eiist/docs/old/vol3no3/article3/v3n3a3.pdf

Downs, Elizabeth, Carlson, Randal D., Repman, Judi y Clark, Kenneth (1999). Web-Based Instructions: Focus on Learning. Recuperado el 21 de febrero de 2002, de ERIC $\begin{array}{llllll}\text { Documento } & \text { IR } & 019 & 584 & \text { / } & \text { ED432254 }\end{array}$ 
http://search.epnet.com/login.aspx?direct=true\&db=eric\&an=ED432254\&loginpag $\underline{\mathrm{e}=\text { login } . \text { asp\&site}=e h o s t}$

Dutton, John, Dutton, Marilyn y Perry, Jo. (2002). How do online students differ from lecture students? Recuperado el 29 de septiembre de 2004, de http://www.sloanc.org/publications/jaln/v6n1/index.asp

Fredericksen, Eric, Pickett, Alexandra, Pelz, William, Swan, Karen, Shea, Peter. (1999). Student satisfaction and perceived learning with on-line courses: Principles and examples from the SUNY Learning Network. Recuperado el 10 de octubre de 2004, de http://www.sloan-

c.org/conference/proceedings/1999Summer/papers/99summer fredericksen2.pdf

Geith, Christine y Vignare, Karen. (2000). Online Degree Programs: Service and Cost. Recuperado el 2 de junio de 2002, de http://www.rit.edu/ 609www/ch/faculty/CostStudy.PDF.pdf

Hughes, Jenny y Attwell, Graham. (2003). A framework for the evaluation of e-learning. Recuperado el 29 de septiembre de 2004, de http://www.theknownet.com/ict smes seminars/papers/Hughes Attwell.html

Kaminski, Karen y Milheim, William D. (2002) Institutional challenges in the creation and delivery of an online degree program. Recuperado el 25 de agosto de 2004, de http://ts. mivu.org/default.asp?show=article\&id=929.

Karsenti, Thierry, Larose, François y Nuñez, Mauricio. (2002). La apertura universitaria a los espacios de formación virtual: Un reto a la autonomía estudiantil. Recuperado el 7 de mayo de 2003, de http://redie.ens.uabc.mx/vol4no1/contenido-karsenti.html

Kassop, Mark. (2003) Ten ways online education matches, or surpasses, face-to-face learning. Recuperado el 25 de agosto de 2004, de http://ts.mivu.org/default.asp?show=article\&id=1059

Katzowitz, Ellen C. y Hall, Helen C. (2003). Predominant learning styles and multiple intelligences of postsecondary allied health students. Recuperado el 20 de febrero de 2006, de http://www.acteonline.org/professional development/handouts/upload/PredominantLearning-Styles-and-Multiple-Intelligences-of-Postsecondary-Allied-Health-Students.doc

Kupritz, Virginia W. (2000). The medium is the message: Implications for teaching in cyberspace. Recuperado el 3 de agosto de2004, de http://www.usq.edu.au/electpub/eiist/docs/old/vol3no2/article2/v3n2a2.pdf

Lavigne. G. (2001). La tâche des professeurs à la Télé-université. Trabajo presentado en la Conférence sur l'enseignement en ligne. Montréal:CAUT/ACPPU.

Levy, Suzanne. (2003). Six factors to consider when planning online distance learning programs in higher education. Recuperado el 26 de agosto de 2004, de http://www.westga.edu/\%7Edistance/ojdla/spring61/levy61.htm 
Mac Keogh, Kay. (2003). Student perceptions of the use of ICTs in European education: Report of a Survey. Recuperado el 11 de junio de 2004, de http://www.oscail.ie/McKeogh.pdf

Mattelard, Armand (1997) Utopía y realidades del vínculo global. Para una crítica del tecnoglobalismo. Recuperado el 22 de febrero 2006, de http://www.felafacs.org/files/mattelart.pdf

McLaren, Peter y Farahmandpur, Ramin (2001) Teaching against Globalization and the New Imperialism: Toward a Revolutionary Pedagogy Recuperado el 22 de febrero 2006, de http://www.gseis.ucla.edu/faculty/pages/mclaren/

Picciano, Anthony G. (2002). Beyond student perceptions: Issues of interaction, presence, and performance in an online course. Recuperado el 21 de febrero de 2006, de http://www.sloan-c.org/publications/jaln/v6n1/pdf/v6n1 picciano.pdf

Pouts-Lajus, Serge. (2002). Trois raisons de dire e-learning. Recuperado el 29 de septiembre de 2004,

http://www. elearningeuropa.info/doc.php? Ing $=3 \& i d=581 \&$ doclng $=3$

Rubio, María J. (2003). Enfoques y modelos de evaluación del e-learning. Recuperado el 29 de septiembre de 2004, de http://www.uv.es/RELIEVE/v9n2/RELIEVEv9n2 1.htm

Schiller, Herbert I. (1998) Conclusions Recuperado el 22 de febrero 2006, de http://commposite.uqam.ca/videaz/docs/hescen.html

Sénécal, Michel (2002) L'autre versant de la mondialisation: démocratiser pour diversifier? Recuperado el 22 de febrero 2006, de http://www.er.uqam.ca/nobel/gricis/actes/panam/Senecalp.pdf\#search='S\%E9n\%E9cal\% $\underline{\underline{20 M i c h e l '}}$

Sierra Caballero, Francisco (1999) Comunicación educativa y economía política: Apuntes sobre políticas culturales e innovación tecnológica Recuperado el 22 de febrero 2006, de http://www.ull.es/publicaciones/latina/ambitos/2/sierra.html

SPOT PLUS. (2004). Survey on students' perspective on technology in teaching and learning in European universities. Recuperado el 6 de octubre de 2004, de http://www.spotplus.odl.org/questionnaire/online quest html

Thormann, Joan. (1999). Rewards and regrets: An online technology in education master's degree program. Accesible en ERIC Documento IR 019584 / ED432253). Recuperado el 21 de febrero de 2006, de http://search.epnet.com/login.aspx?direct=true\&db=eric\&an=ED432253\&loginpage=login. asp\&site=ehost

Twigg, Carol A. (2001). Innovations in online learning: Moving beyond no significant difference. Recuperado el 31 de agosto de 2004, de http://www.thencat.org/Monographs//mpLearn.html 
Western Interstate Commission for Higher Education. (1997). Good practices in distance education. Boulder, $\quad \mathrm{CO}$ : WICHE. Accesible de http://www. wiche.edu/pubs/cart subj.asp?pub id=135

Young, Jeffrey R. (2000). Distance and Classroom Education Seen as Equally Effective. Recuperado el 10 de junio de 2004, de http://chronicle.com/free/v46/i24/24a05501.htm

Young, Jeffrey R. (2002). Hybrid' teaching seeks to end the divide between traditional and online instruction. Recuperado el 30 de septiembre 2004, de http://chronicle.com/free/v48/i28/28a03301.htm

Zemsky, Robert y Massy, William F. (2004). Thwarted innovation. What happened to elearning and why. Recuperado el 11 de enero 2004, de http://www.irhe.upenn.edu/Docs/J un2004/Thwartedlnnovation.pdf 